\title{
Resonance Fluorescence of Fused Silica by the Depopulation of the Ground State
}

\author{
Fuat Bayrakceken $^{1}$ and Korkut Yegin ${ }^{2}$ \\ ${ }^{1}$ Department of Biomedical Engineering, Yeditepe University, Istanbul, Turkey \\ ${ }^{2}$ Department of Electrical and Electronics Engineering, Yeditepe University, Istanbul, Turkey
}

Correspondence should be addressed to Korkut Yegin, kyegin@yeditepe.edu.tr

Received 30 May 2012; Accepted 10 October 2012

Academic Editor: Ipek Karaaslan

Copyright ( 92012 F. Bayrakceken and K. Yegin. This is an open access article distributed under the Creative Commons Attribution License, which permits unrestricted use, distribution, and reproduction in any medium, provided the original work is properly cited.

Spectroscopically pure fused silica has been used in many applications ranging from optoelectronics and optical fibers to laser flash spectroscopy. Although ultraviolet light irradiated optical absorption spectra and coherence fluorescence of silicon dioxide have been studied in the past, we present discrete absorption and resonance coherent fluorescence line of silicon dioxide which were recorded photographically at $288.2 \mathrm{~nm}$. This discrete fluorescence is observed at room temperature using high photon flux $\left(10^{24}\right.$ photon/pulse) excitation spectroscopy.

\section{Introduction}

Resonance fluorescence occurs when atoms/molecules absorb and reemit radiation at the same wavelength. Resonance fluorescence line corresponds to the transition between an electronic excited state and the ground state. The wavelengths of the absorption $\left(\lambda_{A}\right)$ and fluorescence $\left(\lambda_{F}\right)$ are the same for resonance transitions. Resonance fluorescence process of silicon dioxide is shown in Figure 1.

Coherent resonance fluorescence and ultraviolet (UV) light induced optical absorption spectra were reported in [1] and resonance fluorescence was observed in the 250-255 nm band at room temperature by high photon flux excitation spectroscopy. As discussed in previous works [1-7], fused silica exhibits coherent fluorescence in the ultraviolet $\mathrm{C}$ region (UV-C).

UV grade fused silica is an amorphous form of silicon dioxide made from flame hydrolysis of silicon tetrachloride. On top of high UV transmission, it has the properties of low thermal expansion coefficient and high laser damage threshold. Amorphous fused silica form bonds in definite vectorial positions in space such that ring structures that connect molecules can exist $[8,9]$. A random network of atoms in fused silica was shown in [10] and reproduced in Figure 2. Ring structures consist of tetrahedral atomic arrangements and most ring structures contain five or six ring members simply because the bond angle between $\mathrm{O}-\mathrm{Si}-$ $\mathrm{O}$ permits formation of almost perfect tetrahedrons with less strain energy.

The vibrational level patterns for silicon dioxide are not complex for six- or eight rings, which, in turn, enables one to observe transitions in 250-255 $\mathrm{nm}$ band as discrete narrow bands [1]. The bandwidths of these coherent emissions were reported approximately $1 / 25 \mathrm{~nm}$ in [1]. These narrow absorption and emission lines are reproduced from [1] with the permission Bayrakçeken and shown in Figure 3.

Apart from these reported discrete absorption and fluorescence of fused silica, a single line at $288.2 \mathrm{~nm}$ without any vibrational and/or rotational bands was also observed in our experiments and this is shown in Figure 4.

This discrete line at $288.2 \mathrm{~nm}$ was observed previouslyand investigated in the context of Raman resonance scattering and it was stated that only one absorption band was observed at $288.2 \mathrm{~nm}$, seven anti-Stokes lines at 285$288.2 \mathrm{~nm}$ and nine Stokes lines at $288.2-290 \mathrm{~nm}$ band [3]. However, in our experiments, we observed a singleline coherent emission although the UV irradiation was performed on a molecule. This may be due to different 


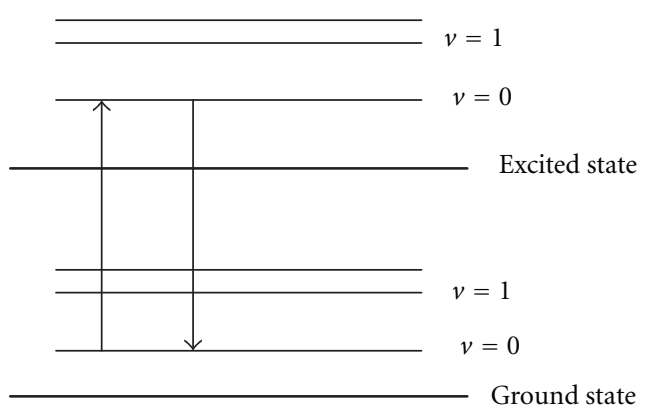

Figure 1: Resonance fluorescence of $\mathrm{SiO}_{2}$.

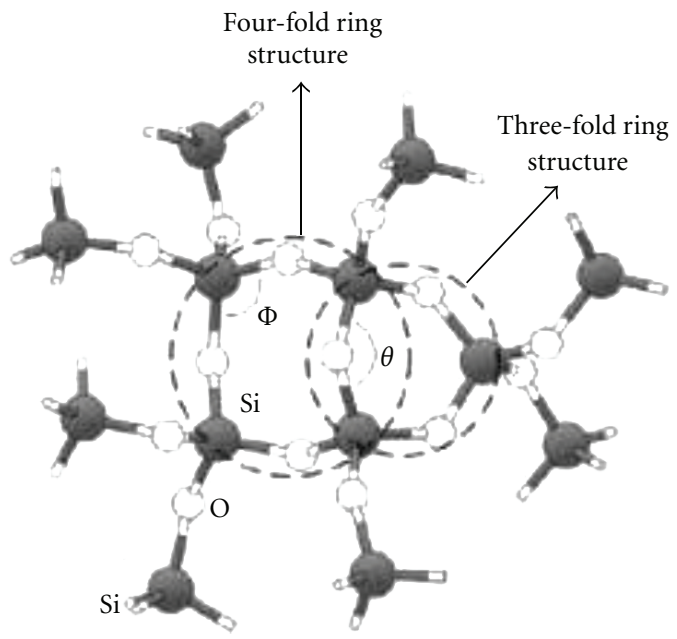

FIGURE 2: Ball and stick model of fused silica in random network (encircled are three-fold and four-fold ring structures) (reproduced from [10]).

photon flux densities used in the studies. The photographic image of the resonance fluorescence line shown in Figure 4 was recorded for the first time.

\section{Material and Methods}

Spectroscopically pure fused silica (Corning Glass Co.), reagent-grade material, was selected for absorption and luminescence experiments. A flash photolysis setup consisting of two parallel phototubes in series, contained in a reflector (front surface mirrored, reflecting the 200$700 \mathrm{~nm}$ band of electromagnetic radiation), was used for the excitation of fused silica sample. The entire optical pumping cavity was flushed with oxygen-free nitrogen to eliminate the presence of paramagnetic oxygen in the optical pumping system. Photoflash energies in the range of 780$1125 \mathrm{~J}$ were used and the flash duration time (1/e time) of the optical pumping device was $2 \mu$ s. Hilger medium quartz spectrograph with slit width $0.025 \mathrm{~mm}$ was used for the recordings of absorption, emission, and lasing spectra. Ilford XK fast blue sensitive plates from Kodak, sensitized with sodium salicylate for UV-C region recordings were developed in Ilford PQ universal developer. Joyce-Loebel MKIIB double-beam recording mirror densitometer was

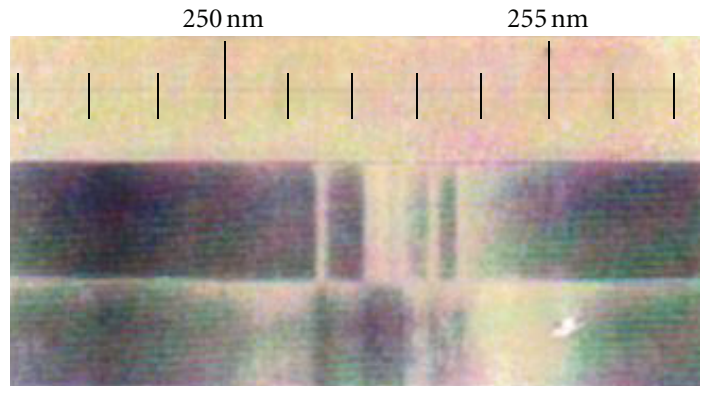

FIgURE 3: Optical discrete absorption and resonance fluorescence of fused silica in the UV (reproduced from [1]).

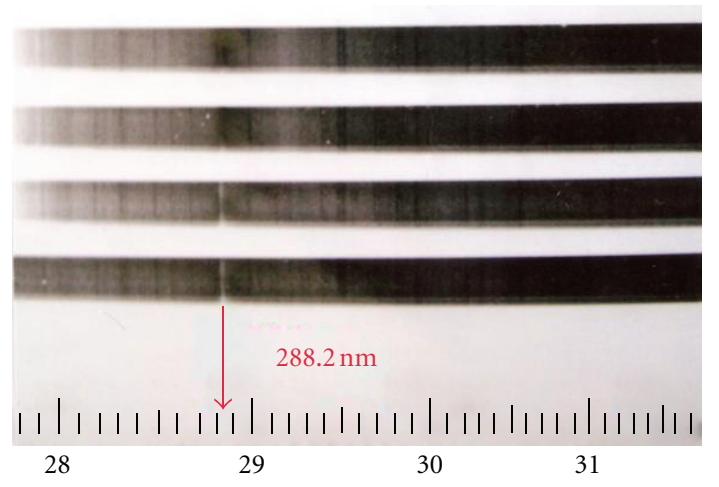

Figure 4: Discrete absorption and resonance fluorescence lines of silicon dioxide at $288.2 \mathrm{~nm}$.

used for photometered spectra. Single flashes were utilized for all spectroscopic recordings.

\section{Results and Discussion}

It is well known that absorption of UV light in the UV-C raises fused silica from the ground state to several excited states. At room temperature, most of the molecules are in the lowest vibrational level of the ground state $(v=0)$ and transitions to higher levels will take place with the absorption of UV light. For silicon dioxide, the vibrational levels are not complex. In fact, symmetric stretching, symmetric bending, and antisymmetric stretching are the only normal modes of $\mathrm{SiO}_{2}$ and these can be observed in the infrared region because electric dipole transitions occur only for normal modes and they are infrared active. The infrared absorption band of $\mathrm{SiO}_{2}$ has been extensively studied in the past $[11,12]$. In the UV region of the spectrum, the interaction of the electromagnetic radiation with electrons in $\mathrm{Si}-\mathrm{O}$ bonds, structural imperfections, Si-Si bonds cause strong absorption [1315]. These lead to sharp UV cut-off around $160 \mathrm{~nm} \mathrm{[12-}$ 15]. The location of the absorption edge naturally depends on the composition, impurity level, structural defects, and temperature. Experimental spectroscopic data between 90 and $350 \mathrm{~nm}$ have been presented in [14].

In general, the bandwidths of normal (prompt) fluorescence are narrower than the corresponding absorption bands due to the dispersion associated with the structure 
during emission. However, if the fluorescence emissions are coherent, the integrated areas under the absorption bands and coherent super-imposed fluorescence emissions will become equal, which, in turn, enables full depopulation of ground state, and all emission transitions will be resonance fluorescence as shown in Figures 3 and 4. The fluorescence radiance can be expressed as follows:

$$
B_{F}=\frac{l}{4 \pi} Y_{21} E_{v_{12}} \int_{0}^{\infty} k_{v} d v
$$

where $B_{F}$ is absolute radiance $l$ is the path length, $Y_{21}$ is power efficiency (due to quenching) $W_{\text {fluorescenced }} / W_{\text {absorbed }}, E_{v_{12}}$ is the spectral irradiation of source at absorption line frequency of $v_{12}$, and $k_{v}$ is the absorption coefficient. The integration term provides the integrated absorption coefficient over the absorption line, which is a function of the concentration of ground and excited states.

\section{Conclusion}

We note that no additional fluorescence and/or scattering were observed in lower bands, that is, $270-275 \mathrm{~nm}$. Moreover, all spectral and lasing emissions were recorded at room temperature. Therefore, we also argue that the population inversion and the depopulation of ground state are permissible. As shown in Figure 4, resonance fluorescence at $288.2 \mathrm{~nm}$ was very intense and coherent. Thus, it is possible to use fused silica as a laser material in the UV or as a UV detector with its inherit visible-blind property.

\section{References}

[1] F. Bayrakçeken, "Sensitive detection of optical discrete absorption and lasing of fused silica by the depopulation of the ground state," Applied Physics B, vol. 105, pp. 573-574, 2011.

[2] F. Bayrakceken, A. Yaman, and B. B. Jomehri, "Resonance fluorescence studies of spectroscopically pure $\mathrm{SiO}_{2}$," Journal of the Indian Chemical Society, vol. 77, no. 9, pp. 445-446, 2000.

[3] A. Yaman, A. B. Bayrakçeken, O. J. Demir, and F. Bayrakçeken, "Electronic absorption and Raman resonance scattering of spectroscopically pure $\mathrm{SiO}_{2}$," Spectrochimica Acta A, vol. 56, no. 10, pp. 1901-1903, 2000.

[4] S. Hayashi and K. Yamamoto, "Optical properties of Si-rich $\mathrm{SiO}_{2}$ films in relation with embedded Si mesoscopic particles," Journal of Luminescence, vol. 70, pp. 352-363, 1996.

[5] Y. Sakurai and K. Nagasawa, "Green photoluminescence band in $\gamma$-irradiated oxygen-surplus silica glass," Journal of Applied Physics, vol. 86, article 1377, 5 pages, 1999.

[6] A. Teramoto, K. Kobayashi, Y. Ohno, and A. Shigetomi, "Excess currents induced by hot hole injection and FN electron injection in thin $\mathrm{SiO}_{2}$ films," IEEE Transactions on Electron Devices, vol. 48, no. 5, pp. 868-873, 2001.

[7] A. Anedda, C. M. Carbonaro, R. Corpino, and A. Serpi, "Vacuum ultraviolet absorption of silica samples," Journal of Non-Crystalline Solids, vol. 245, pp. 183-189, 1999.

[8] S. V. King, "Ring configurations in a random network model of vitreous silica," Nature, vol. 213, no. 5081, pp. 1112-1113, 1967.

[9] J. P. Reno and I. Ebbsjo, "Structure of rings in virteous $\mathrm{SiO}_{2}$," Physical Review B, vol. 47, pp. 3053-3062, 1993.
[10] S. Vukelic, S. Ryu, B. Gao, and Y. L. Yao, "Structural modification of amorphous fused silica under femtosecond laser irradiation," in Proceedings of the ASME International Manufacturing Science and Engineering Conference (MSEC '08), pp. 227-236, October 2008.

[11] A. M. Efimov, Optical Constants of Inorganic Glasses, CRC Press, Boca Raton, Fla, USA, 1995.

[12] I. Fanderlik, Optical Properties of Glass, vol. 5 of Glass Science and Technology, Elsevier Science, New York, NY, USA, 1983.

[13] K. Kajihara, "Improvement of vacuum-ultraviolet transparency of silica glass by modification of point defects," Journal of the Ceramic Society of Japan, vol. 115, no. 1338, pp. 85-91, 2007.

[14] G. H. Siegel, "Ultraviolet spectra of silicate glasses: a review of some experimental evidence," Journal of Non-Crystalline Solids, vol. 13, no. 3, pp. 372-398, 1974.

[15] N. Shimodaira, K. Saito, A. J. Ikushima, T. Kamihori, and S. Yoshizawa, "VUV transmittance of fused silica glass influenced by thermal disorder," in Proceedings of the 13th Optical Microlithography, vol. 4000 of Proceedings of SPIE, pp. 15531559, March 2000. 


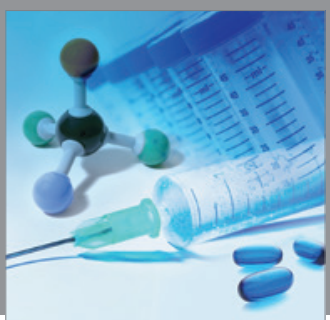

International Journal of

Medicinal Chemistry

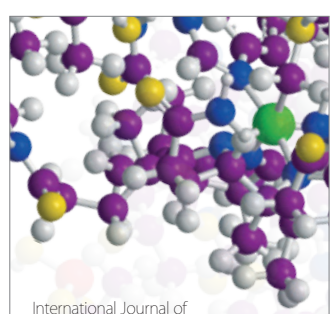

Carbohydrate Chemistry

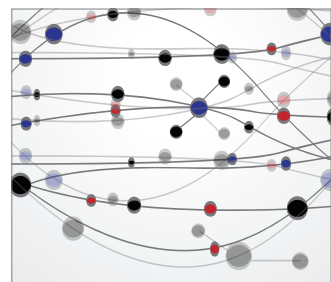

The Scientific World Journal
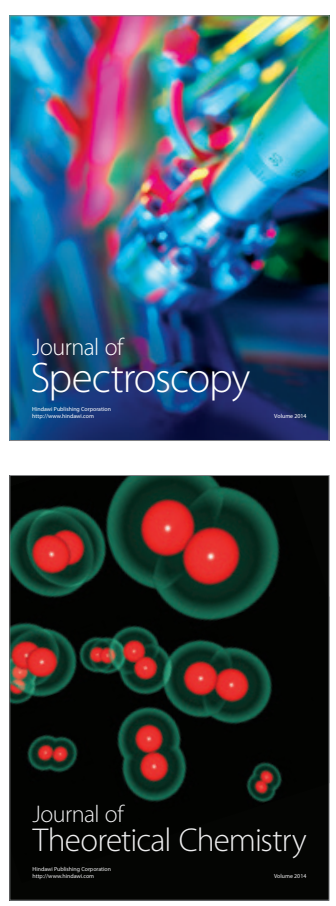
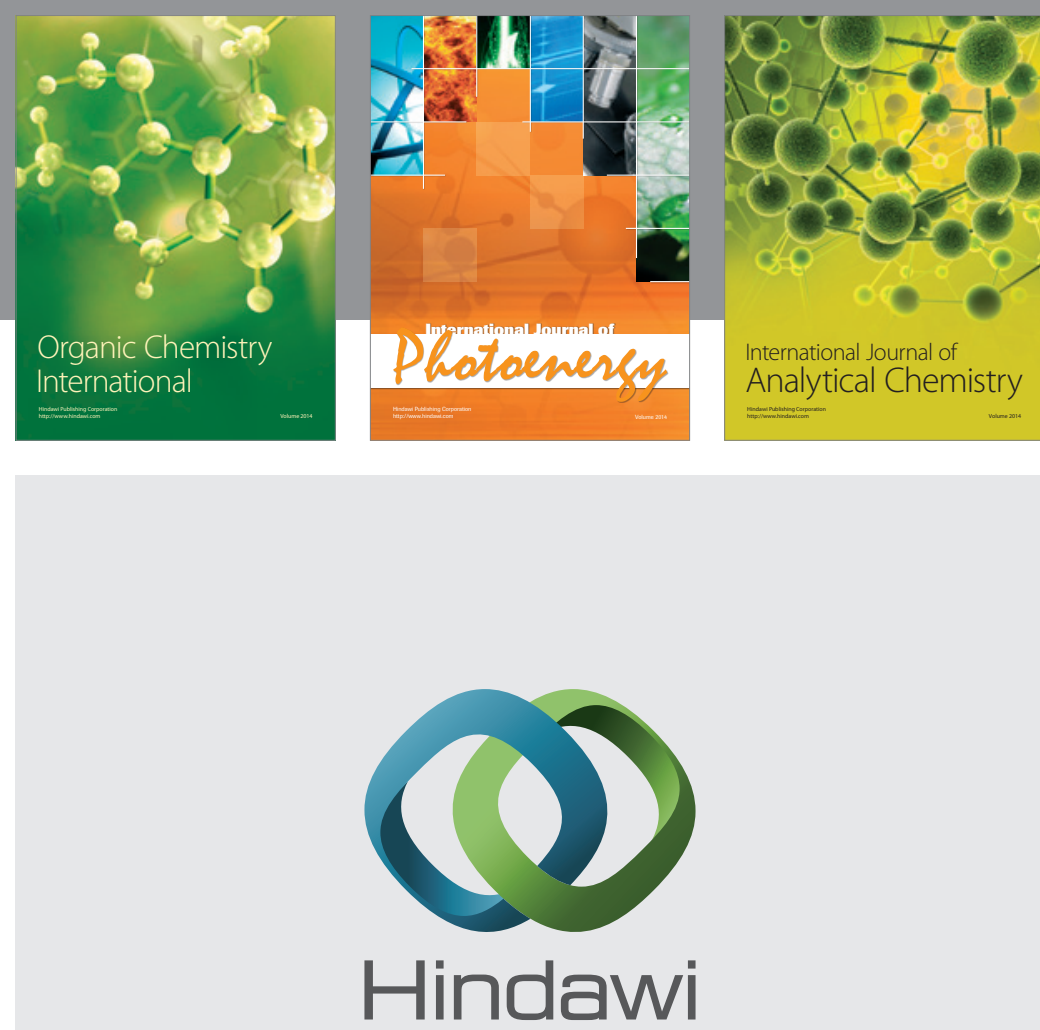

Submit your manuscripts at

http://www.hindawi.com
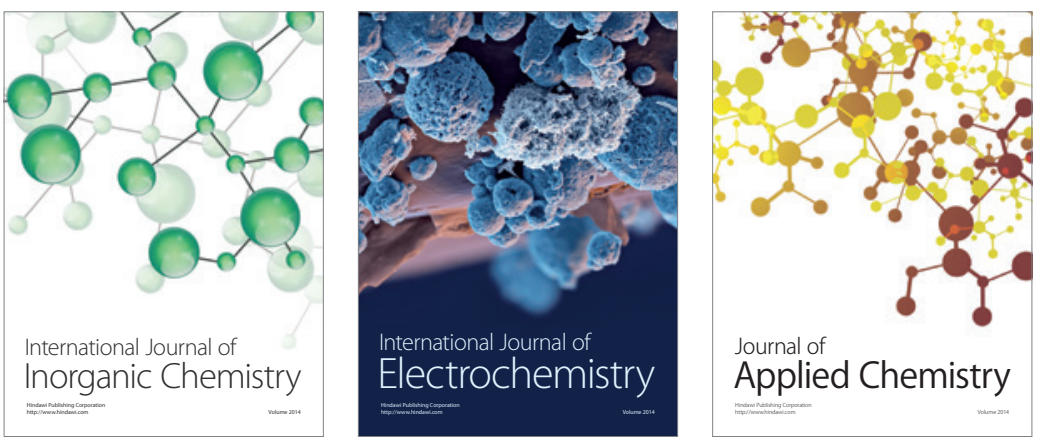

Journal of

Applied Chemistry
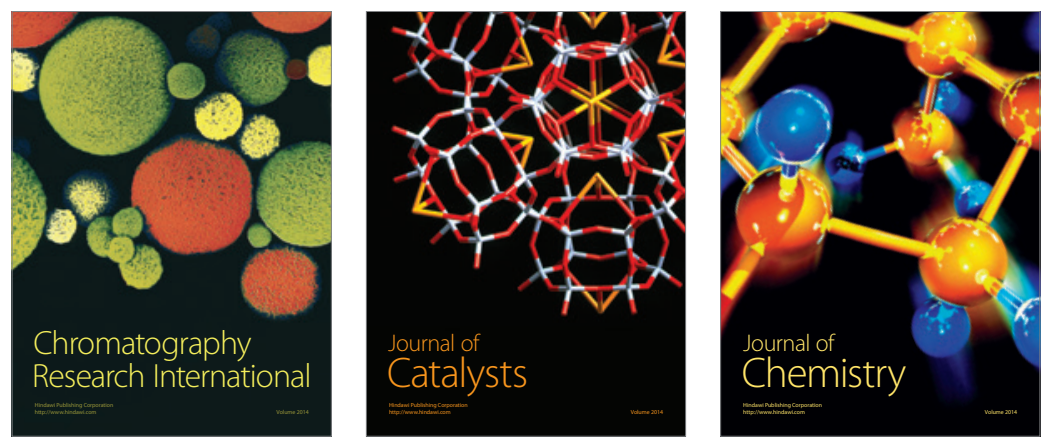
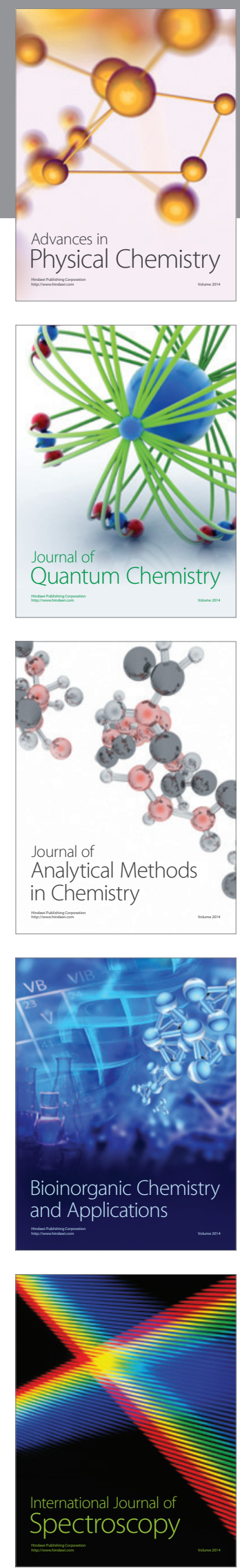